\title{
评述
}

\section{微生态制剂对断奶仔猪肠道健康的影响 及作用机制}

王天威 ${ }^{1,2 \dagger}$, 滕坤玲 ${ }^{1 \dagger}$, 刘刚 ${ }^{3}$, 刘亚勇 ${ }^{1,2}$, 张欣 ${ }^{4}$, 董恩球 ${ }^{4}$, 印遇龙 ${ }^{3}$, 钟瑾 ${ }^{1,2 *}$

1. 中国科学院微生物研究所, 北京 100101 ;

2. 中国科学院大学, 北京 100101 ;

3. 中国科学院亚热带农业生态研究所, 长沙 410125;

4. 龙大食品集团有限公司, 烟台 265231

$\dagger$ 同等贡献

* 联系人, E-mail: zhongj@im.ac.cn

收稿日期：2018-07-16; 接受日期：2018-10-20; 网络版发表日期：2019-01-09

摘要断奶仔猪腹泻造成仔猪高死亡率是困扰养猪业发展的重要问题. 幼龄仔猪肠道屏障和免疫系统都尚未发 育成熟，容易受到病原菌、病毒、应激等因素的影响导致仔猪胃肠道功能紊乱，诱发仔猪断奶综合征。抗生素等 药物虽然在一定程度上可以改善养殖现状, 减少经济损失, 但抗生素滥用以及由此产生的细菌耐药性问题已严重 影响环境及人体健康, 成为全球性的重大问题, 所以研究安全环保的抗菌药物, 以减少或替代抗生素的使用迫在 眉睥. 微生态制剂具有安全、无毒副作用、不易产生耐药性、可调节动物肠道微生态等特点, 因此, 了解微生态 制剂如何改善肠道健康及其中的作用机制, 对有效利用它们来改善仔猪腹泻具有十分重要的意义. 本文对断奶仔 猪腹泻的原因和微生态制剂在仔猪中发挥益生功效的主要作用机制进行了综述.

关键词微生态制剂，断奶仔猪，肠道健康，作用机制

断奶仔猪腹泻是造成仔猪高死亡率的重要原因, 其发病率和死亡率都相对较高(发病率为 $50 \%$ $80 \%$, 病 死率高达 $80 \%)^{[1]}$, 已经成为困扰养猪业的重要问题. 仔 猪腹泻的原因较为复杂, 主要为感染性腹泻, 包括病毒 性感染(如猪传染性胃肠炎病毒(transmissible gastroenteritis virus, TGEV)、猪流行性腹泻病毒(porcine epidemic diarrhea virus, PEDV)和猪轮状病毒(rotavirus, $\mathrm{RV})$ ), 细菌性感染(如产肠毒素大肠杆菌(enterotoxigenic E.coli, ETEC), 产志贺毒素大肠杆菌(shiga toxin- producing E. coli, STEC)和黏附与脱落性大肠杆菌(attaching-effacing E. coli, AEEC))和寄生虫感染(如棘头 虫、猪球虫、隐狍子虫等 $)^{[1,2]}$. 仔猪腹泻可导致仔猪 成活率下降、生长缓慢、发育停滞而成为僵猪, 甚至 发生死亡, 这给生猪产业造成重大的经济损失. 无论 何种原因造成的仔猪腹泻均与肠道健康息息相关. 预 防和治疗仔猪腹泻的方式多种多样, 包括抗生素(金霉 素、硫酸安普霉素、诺氟沙星、环丙沙星、恩诺沙星 等)、微生态制剂(益生元、益生菌和合生元)、氧化

引用格式: 王天威, 滕坤玲, 刘刚, 等. 微生态制剂对断奶仔猪肠道健康的影响及作用机制. 中国科学: 生命科学, 2019, 49: 97-107 Wang T W, Teng K L, Liu G, et al. The functions and mechanisms of microecological agents in weaning piglet intestine (in Chinese). Sci Sin Vitae, 2019, 49: 97-107, doi: 10.1360/N052018-00143 
锌、复方中草药、植物精油、溶菌酶、抗菌肽、酶制 剂、酸化剂和中链脂肪酸等. 在实际生产应用中使用 最为广泛的主要是抗生素和氧化锌等化学制剂, 但是 长期使用抗生素会造成动物源和食物源病原菌和共生 菌的耐药菌株出现, 影响肠道菌群平衡 ${ }^{[3,4]}$, 氧化锌也 会对土壤生态系统造成危害，同时诱导家畜相关耐甲 西林金黄色葡萄球菌(livestock-associated methicillinresistant Staphylococcus aureus, LAMRSA)产生 ${ }^{[5]}$. 随 着全球经济一体化地不断发展，中国畜牧业的相关标 准与法律法规必然要与发达国家相接轨, 因此“无抗低 锌”的绿色养殖方式必将成为养猪业未来的发展趋势。 微生态制剂具有促进仔猪生长性能、改善腹泻、提高 免疫能力和调节肠道菌群等益生功能, 已广泛应用在 仔猪饲粮中. 本文主要针对仔猪腹泻原因及微生态制 剂改善仔猪肠道健康的机制研究进行综述.

\section{1 断奶仔猪腹泻原因}

在现代化养猪业生产中, 仔猪通常在3 4周断奶, 有的仔猪甚至在1 2周就进行断奶，仔猪的日粮由断 奶前富含乳糖、脂肪和酪蛋白的母乳变为含有大量淀 粉、来源各异的蛋白质及脂肪的固体饲料，导致仔猪 食量下降，小肠线毛/隐窝深度降低，营养物质吸收严 重降低, 生产性能下降, 引起应激反应 ${ }^{[6,7]}$. 断奶时, 仔 猪的胃肠消化酶包括胃蛋白酶、胰淀粉酶、脂酶、胰 蛋白酶和糜蛋白酶等分泌不足, 活性急剧下降, 需两周 才能恢复至断奶前水平. 因此, 饲料无法被顺利地消化 吸收, 在后肠段发生腐败分解, 引起大肠杆菌等有害菌 群增殖，产生很多胺类毒性物质，对肠壁组织造成损 伤，使肠道蠕动加快导致腹泻 ${ }^{[8]}$. 同时，仔猪断奶后与 母猪分开, 进行混窝生活, 生活环境急剧变化引起仔猪 吵闹、斗争、心脏增速、分泌压力相关因子等，也使 仔猪产生应激反应，从而发生腹泻 ${ }^{[6,9]}$. 仔猪在断奶期 间还有可能发生肠道炎症响应相关途径激活，激素活 力改变, 胃部运动降低, 从而引起肠道萎缩, 降低线毛 高度，降低营养、体液和电解质的吸收、增加抗原和 毒素的渗透性等 ${ }^{[10]}$. Lallès等人 ${ }^{[11]}$ 认为无论饮食改变 或者环境改变都会导致仔猪肠道微生物改变，而微生 物改变与断奶后仔猪肠道生态环境和肠道感染有关.

猪肠道微生态是复杂的生态系统 ${ }^{[12]}$ ，其构成和多 样性都随着生长时期和肠道不同部位有所变化. Petri
等人 ${ }^{[13]}$ 发现仔猪出生 1 天肠道微生物以大肠杆菌和链 球菌为主, 出生3 20天乳酸菌逐渐成为优势菌种. Gresse等人 ${ }^{[10]}$ 推测可能是大肠杆菌和链球菌等好氧菌 消耗氧气, 为拟杆菌(Bacteroides)、乳杆菌(Lactobacillus)、双歧杆菌(Bifidobacterium)和梭菌(Clostridium) 等创造了适宜生存的厌氧环境, 供其定殖. Bian等人 ${ }^{[14]}$ 研究表明母乳喂养能够保持肠道微生态平衡, 断奶造 成仔猪肠道菌群结构发生改变, 其中乳杆菌属数量降 低, 梭菌属、普氏菌属(Platts species)和兼性厌氧的变 形杆菌属等增加, 仔猪肠道微生态遭到破坏, 即微生态 失调(dysbiosis) ${ }^{[7,10]}$. 微生态失调的代表特征就是专性 厌氧菌显著降低，兼性厌氧菌的数量相对增加，从而 导致肠道紊乱和胃肠道感染. 黏膜层是保护肠道表皮 完整的重要屏障, 抗生素通常作为断奶仔猪的保健药 物, 但是抗生素会导致肠道微生物的多样性和稳定性 降低，宿主释放的唾液酸和微生物发酵产生的琥珀酸 增加使得艰难梭菌和沙门氏菌(Salmonella)等病原菌 迅速增殖，增加了肠道被病原菌感染的几率 ${ }^{[15]}$. 同时， 肠道表皮通透性增加甚至失去完整性, 更有利于病原 菌及其释放毒素的入侵.

仔猪病毒性腹泻病死率高、传播速度快、造成经 济损失严重. 导致仔猪病毒性腹泻的主要有猪轮状病 毒(rotavirus，RV)、猪传染性胃肠炎病毒(transmissible gastroenteritis virus, TGEV)、猪流行性腹泻病毒(porcine epidemic diarrhea virus, PEDV)以及最新发现的猪 急性腹泻综合征冠状病毒(swine acute diarrhoea syndrome coronavirus, SADS-CoV) ${ }^{[16]}$. PEDV最为常见, 属 于正链RNA病毒, 具有侵染性. PEDV的主要结构包括 刺突糖蛋白(the spike glycoprotein, S; 150 200 kD)、 整合膜蛋白(the integral membrane glycoprotein, M; 20 30 kD)、核衣壳磷蛋白(the nucleocapsid phosphoprotein, $\mathrm{N} ; 43 \sim 50 \mathrm{kD})^{[17]}$. S 蛋白可以在宿主体内作为一 种免疫蛋白介导中和产生的对应抗体，作为表面抗原， 可以识别靶细胞, 使细胞表面受体和病毒粒子结合, 然 后侵入宿主细胞，刺激机体产生抗体， $\mathrm{S}$ 蛋白的大小会 随分离株的地区差异而有所不同 ${ }^{[17]}$. M蛋白是构成病 毒粒子的关键部分, 能够促进病毒粒子的重装, 介导 产生抗体通过补体作用中和病毒，还可促进机体产生 $\alpha$ 干扰素. N蛋白既是PEDV的保守结构蛋白又是抗原 之一, 编码 441 个氨基酸, 与病毒的复制和转录有一定 的关系, 具有免疫原性, 在刺激机体的细胞免疫和体液 
免疫方面发挥着非常重要的作用 ${ }^{[17]}$. PEDV感染的病 猪表现为呕吐、腹泻和脱水，而且年龄越小，症状越 重, 甚至死亡. PEDV在自然情况下, 猪群都比较易感, 该病的传染源主要是粪便和带毒猪. PDEV存在明显 的季节性, 流行主要发生在夏季和冬季 ${ }^{[1,18]}$.

造成仔猪细菌性腹泻的致病菌种类较多, 其中两 个最主要的病原菌是大肠杆菌和鼠伤寒沙门氏菌 $(\mathrm{Sal}$ monella enterica serovar typhimurium $)^{[19]}$. 致病性大肠 杆菌，如产肠毒素大肠杆菌(enterotoxigenic E. coli, ETEC)是仔猪断奶后感染的主要病原, 主要通过其表 面的黏附素吸附于小肠上皮细胞，然后产生热敏肠毒 素(heat labile enterotoxin, LT)和/或耐热肠毒素(heat stable enterotoxin, ST). 产志贺毒素大肠杆菌(Shiga toxin-producing E. coli, STEC)主要通过黏附素在消化 道定殖, 产生志贺样毒素(Stx2e, 又称Vero细胞毒素), 或者同时产生ST和LT而引起断奶仔猪腹泻和/或水肿 病. 黏附与脱落性大肠杆菌(attaching-effacing E.coli, AEEC)主要通过细菌外膜蛋白紧密素(intimin)紧密局 灶性黏附于小肠上皮细胞, 引起黏附与脱落(attaching and effacing, $\mathrm{A} / \mathrm{E}$ )效应，其大多在回肠部引起轻度或 中度炎症，菌落多聚集于十二指肠和盲肠间，造成小 肠轻度至中度线毛萎缩 ${ }^{[2]}$. 鼠伤寒沙门氏菌是仔猪断 奶后感染的另外一个主要病原, 它利用类注射器的三 型分泌系统(T3SS)识别并黏附到宿主肠道细胞 ${ }^{[20]}$, 从 而造成肠道细胞微线毛和细胞骨架改变, 诱导宿主细 胞超微结构损伤, 影响肠道吸收, 导致仔猪腹泻, 失去 生长性能.

兽用抗生素虽然在一定程度上能够抑制一些致病 菌, 防治仔猪腹泻, 降低成本, 但是会造成动物源和食 物源的病原菌和共生菌的耐药菌株出现. 具有耐药性 的病原微生物不仅对动物健康造成损害，还会随着食 物链逐渐转移到人体, 从而引起人类健康问题 ${ }^{[21]}$. 由 于抗生素具有广谱的杀菌作用，不仅能够消灭病原菌， 同时也会对肠道益生菌和共生菌造成破坏。临床统计 表明，由于使用抗生素造成腹泻的病人多达 $30 \%{ }^{[22]}$. 随着人与人之间的接触更会造成共生菌的耐药性菌株 大肆传播，引起全球生态问题. 我国农业部已禁止4种 人兽共用抗生素用于食品动物, 并深入实施《全国遏 制动物源细菌耐药行动计划(2017 2020年)》. 因此, 研发安全高效的抗生素替代品来增强仔猪肠道的防御 屏障势在必行.

\section{2 微生态制剂分类}

微生态制剂(microecologics)是指能够调整微生态 失调、保持微生态平衡、提高宿主健康水平的益生菌 及其代谢产物和促进肠道益生菌生长的制品，是比较 理想的抗生素替代品. 微生态制剂主要分为益生菌 (probiotic)、益生元(prebiotic)和合生元(synbiotic) 三类.

\section{1 益生菌}

益生菌(probiotics)一词是由Parker ${ }^{[23]}$ 在1974年首 先提出的, 他将其描述为“有助于肠道微生物平衡的微 生物或物质”, 被美国食品药品监督管理局指定为公认 的安全(generally recognized as safe, GRAS)成分. 目前, 对益生菌的最新阐述是指在摄入适当量时能够对宿主 产生有益作用的一类活的微生物 ${ }^{[24]}$. 益生菌主要以乳 酸菌为主, 如乳杆菌、双歧杆菌、粪肠球菌(Enterococcus faecalis)、片球菌(Pediococcus)、链球菌(Streptococcus) 等, 此外还有芽狍杆菌 (Bacillus) 和酵母菌 (Saccharomyce $)^{\text {等 }}{ }^{[25]}$. 益生菌发挥其功能需要具备以 下特点: (i ) 对宿主有益生作用; (ii) 非致病性, 无毒 副作用; (iii) 菌制剂需要含有足够量的活菌数; (iv) 能 够在胃肠道内定殖和代谢; ( v ) 在储藏和运输过程中 能够保存活力; (vi) 具有良好的适口性; (vii) 从与预期 宿主相同的物种分离 ${ }^{[26]}$. 近年来, 大量研究表明益生 菌可改善仔猪肠道健康, 包括降低有害菌、增强肠道 屏障、增加线毛高度、促进营养吸收、提高日增重、 降低腹泻率等(表1).

\section{2 益生元}

益生元被定义为在肠道中选择性发酵的膳食纤 维, 它允许胃肠道微生物群的组成和/或活性的特异性 改变, 从而对宿主产生有益的生理作用 ${ }^{[38]}$. 益生元大 多数属于非淀粉多糖, 如低聚果糖(scFOS)、低聚半 乳糖(galactooligosaccharides, GOS)、纤维寡糖(cellooligosaccharide)、甘露寡糖(mannanoligosaccharides) 和菊粉(inulin)等. 益生元主要具有增加后肠段短链脂 肪酸(short chain fatty acid, SCFA)浓度, 增加免疫球蛋 白的分泌，降低致病性大肠杆菌、沙门氏菌等功能 (表2). 
表 1 益生菌在仔猪中的作用

Table 1 The effect of probiotics on piglets

\begin{tabular}{|c|c|c|c|c|}
\hline 益生菌 & 剂量 & 仔猪年龄 & 益生菌的功能 & 参考文献 \\
\hline L. reuteri $\mathrm{I} 5007$ & $10^{10} \mathrm{CFU} /$ 头/天 & 4 24天 & $\begin{array}{c}\text { 提高新生仔猪结肠防御肽的表达; 增加结 } \\
\text { 肠丁酸盐浓度; 上调PPAR 和GPR } 41 \text {; 不改 } \\
\text { 变结肠微生物群落结构 }\end{array}$ & [27] \\
\hline L. reuteri $\mathrm{I} 5007$ & $6 \times 10^{9} \mathrm{CFU}$ 头/天 & $2 \sim 16$ 天 & $\begin{array}{l}\text { 增加空肠和回肠表皮细胞紧密连接蛋白 } \\
\text { (claudin-1, occludin和ZO-1)的表达 }\end{array}$ & [28] \\
\hline $\begin{array}{l}\text { Pediococcus acidilactici }(\mathrm{PA}) \text { 和 } \\
\text { Saccharomyces cerevisiae subsp. } \\
\text { boulardii }(\mathrm{SCB})\end{array}$ & $2 \times 10^{9} \mathrm{CFU} / \mathrm{KG}$ & 10 37天 & $\begin{array}{c}\text { PA组: 增加厚壁菌门的丰度; } \\
\text { SCB组: 增加结肠紫单胞菌科和疮微菌科 } \\
\text { 丰度 }\end{array}$ & [29] \\
\hline $\begin{array}{c}\text { L. acidophilus或 Pediococcus } \\
\text { acidilactici }\end{array}$ & $10^{9} \mathrm{CFU} / \mathrm{g}$ 发酵饲料 & 4周 & $\begin{array}{c}\text { 增加粪便中乳酸菌的含量, 降低大肠杆菌 } \\
\text { 的数量; 提高日增重; 提高粪便中乳酸浓 } \\
\text { 度; 降低腹泻率; 增加线毛高度并降低隐 } \\
\text { 窝深度 }\end{array}$ & [30] \\
\hline L. salivarius UCC118 & $10^{10} \mathrm{CFU} /$ 头/天 & 26 天 & $\begin{array}{l}\text { 降低螺旋体门的水平; 增加饲料转换率; } \\
\text { 厚壁菌门和拟杆菌门有略微增加趋势 }\end{array}$ & [31] \\
\hline Enterococcus faecalis LAB31 & $0.5 \sim 2.5 \times 10^{9} \mathrm{CFU} / \mathrm{KG}$ 饲料 & 4 8周 & $\begin{array}{l}\text { 增加日增重和饲料转换率; 降低腹泻指数; } \\
\text { 增加微生物多样性; 增加乳杆菌数量 }\end{array}$ & [32] \\
\hline L. sobrius DSM 16698. & $\begin{array}{c}10^{10} \mathrm{CFU} / \text { 头/天 } 5 \times 10^{10} \mathrm{CFU} \\
\mathrm{ETEC} \mathrm{F} 4\end{array}$ & 3 4周 & $\begin{array}{c}\text { 显著降低回肠和结肠内ETEC负载量; 提 } \\
\text { 高日增重 }\end{array}$ & [33] \\
\hline L. rhamnosus & $\begin{array}{c}10^{11} \mathrm{CFU} / \text { 头/天 } 10^{9} \mathrm{CFU} \\
\mathrm{ETEC} \mathrm{K} 88\end{array}$ & 26 36天 & $\begin{array}{l}\text { 降低大肠杆菌数量; 增加双歧杆菌和乳杆 } \\
\text { 菌数量; 降低腹泻率; 弱化ETEC 引起的血 } \\
\text { 清中IL-6的表达; 增加TNF- } \alpha \text { 的表达 }\end{array}$ & [34] \\
\hline $\begin{array}{l}\text { Bifidobacterium longum subsp. } \\
\text { infantis CECT } 7210 \text { 和 } B \text {. animalis } \\
\text { subsp. lactis BPL6 }\end{array}$ & $\begin{array}{c}10^{9} \mathrm{CFU} / \text { 头/ } / 0^{9} \mathrm{CFU} \text { Salmonella } \\
\text { Typhimurium }\end{array}$ & 4 6周 & $\begin{array}{l}\text { 增加采食量; 降低粪便中沙门氏菌的排出 } \\
\text { 量; 提高线毛/隐窝的比率, 降低腹泻率; 增 } \\
\quad \text { 加回肠乙酸浓度, 降低结肠氨浓度; }\end{array}$ & {$[35]$} \\
\hline Bacillus subtilis和 $B$. lichenformis & $\begin{array}{c}3.9 \times 10^{8} \mathrm{CFU} \text { 或 } 7.8 \times 10^{8} \mathrm{CFU} / \text { 头 } / \text { 天 } 10^{10} \\
\mathrm{CFU} \\
\text { ETEC F4 }\end{array}$ & 36 天 & $\begin{array}{c}\text { 降低粪便中大肠杆菌的丰度; 下调TLP4, } \\
\text { NOD2, iNOS, IL-8和IL-22 }\end{array}$ & [36] \\
\hline Saccharomyces cerevisiae & $\begin{array}{c}5 \times 10^{10} \mathrm{CFU} / \mathrm{KG} \text { 饲料 } 1.5 \times 10^{8} \mathrm{CFU} \\
\text { ETEC F4 }\end{array}$ & 4 7周 & 降低粪便中大肠杆菌ETEC的排出量 & {$[37]$} \\
\hline
\end{tabular}

表 2 益生元在母猪及仔猪中应用

Table 2 The effect of prebiotics on sows and piglets

\begin{tabular}{|c|c|c|c|c|}
\hline 益生元 & 剂量 & 实验对象 & 益生元的功能 & 参考文献 \\
\hline 低聚果糖(scFOS) & $10 \mathrm{~g} /$ 天 & 母猪和其仔猪 & $\begin{array}{l}\text { 初乳的IgA, TGF- } \beta 1 \text { 显著增加; 仔猪潘氏 } \\
\text { 细胞分泌的IFN } \gamma \text { 和SIgA 增加; 仔猪盲肠短 } \\
\text { 链脂肪酸(尤其是丙酸、丁酸和戊酸)增加 }\end{array}$ & [39] \\
\hline $\begin{array}{c}\text { 纤维寡糖 } \\
\text { (cello-oligosacharide) }\end{array}$ & $1.5 \sim 4.5 \mathrm{~g} / \mathrm{kg}$ & 3 5周仔猪 & $\begin{array}{c}\text { 增加乳杆菌; 降低肠杆菌; 增加线毛高度 } \\
\text { 和表面积 }\end{array}$ & [40] \\
\hline $\begin{array}{c}\text { 甘露寡糖 } \\
\text { (mannanoligosaccharides) }\end{array}$ & $0.2 \%$ 饲料 & 3 6周仔猪 & 降低肠杆菌科数量 & [41] \\
\hline 菊粉(inulin) & $3 \%$ 饲料 & 母猪和其仔猪 & $\begin{array}{c}\text { 增加妊娠期和哺乳期母猪粪便中肠球菌 } \\
\text { 的数量; 增加仔猪盲肠内肠球菌数量; 增 } \\
\text { 加仔猪胃内真菌和盲肠中柔嫩梭菌的数 } \\
\text { 量; 降低仔猪胃中肠杆菌和食淀粉乳杆菌 } \\
\text { 的数量 }\end{array}$ & [42] \\
\hline $\begin{array}{c}\text { 低聚果糖 } \\
\text { (fructooligosac charides) }\end{array}$ & $\begin{array}{c}1 \% \text { 水/饲料 } \\
10^{7} \mathrm{CFU} \text { Salmonella Typhimurium }\end{array}$ & 3 7周仔猪 & 降低粪便中沙门氏菌的排除量 & [43] \\
\hline
\end{tabular}




\section{3 合生元}

合生元是指活微生物添加剂(益生菌)与特定底物 (益生元)的组合, 利于促进宿主生长. 由于特定底物容 易被益生菌利用，因此这种组合可以促进益生菌生存， 从而有利于宿主健康 ${ }^{[26]}$. 合生元调节动物肠道健康的 功能主要包括增加营养吸收和短链脂肪酸含量、调节 肠道菌群、提高宿主肠道免疫屏障、下调炎症因子等 (表3). 通常情况下, 合生元中益生菌和益生元具有协 同作用.

\section{3 微生态制剂的作用机制}

微生态制剂调节肠道健康的机制多种多样, 如微 生态制剂可通过优势种群、生物夺氧、生物拮抗、提 供营养、净化胃肠道, 增加胃肠道有益微生物数量, 抑 制有害微生物的生长等以减少动物疾病发生，提高动 物生产性能. 此外, 微生态制剂可刺激机体免疫器官 发育，提高仔猪抗体水平或巨噬细胞活性，增强机体 免疫功能. 归纳起来微生态制剂的作用机制主要包括 以下几个方面.

\section{1调节肠道pH、抑制致病菌增殖和减少毒素 产生}

益生菌可通过产生短链脂肪酸、过氧化氢 $\left(\mathrm{H}_{2} \mathrm{O}_{2}\right)$ 和细菌素等抗菌物质抑制病原菌生长, 降低它们产生
的肠毒素、志贺毒素等毒力因子的表达，促进肠道健 康 ${ }^{[33,34,37]}$. Barba-Vidal等人 ${ }^{[35]}$ 发现饲喂长双歧杆菌( Bifidobacterium longum) 和动物双歧杆菌能够增加回肠 乙酸浓度. Asahara等人 ${ }^{[51]}$ 发现在产生志贺毒素(Stx)的 大肠杆菌O157:H7感染的小鼠(Mus musculus)模型中, 短双歧杆菌(Bifdobacterium breve)产生高浓度的乙酸 $(56 \mathrm{mmol} / \mathrm{L})$, 可降低肠腔 $\mathrm{pH}$ 至6.75. 这种高浓度乙酸 和低 $\mathrm{pH}$ 的肠腔环境不利于大肠杆菌的生长, 抑制 Stx 毒素的产生, 提高动物存活率. $\mathrm{H}_{2} \mathrm{O}_{2}$ 是一种具有抗微 生物活性的化合物，据文献报道益生菌可以通过产生 $\mathrm{H}_{2} \mathrm{O}_{2}$ 抑制病原菌, 如约氏乳杆菌 (Lactobacillus johnso$n i i) \mathrm{NCC} 533$ 具有合成 $\mathrm{H}_{2} \mathrm{O}_{2}$ 的能力, 其发酵液上清可明 显抑制致病菌Salmonella enterica serovar Typhimurium $\mathrm{SL} 1344^{[52]}$. 此外, 益生乳杆菌中许多菌种可以产生细 菌素, 这些细菌素可以抑制其他乳酸杆菌或分类学相 近的革兰氏阳性细菌, 甚至可以抑制酵母菌和霉菌 ${ }^{[53]}$, 如唾液乳杆菌(L. salivarius) UCC118产生小肽, 能够抑 制包括芽孢杆菌、葡萄球菌(Staphylococcus)、肠球菌 (Enterococcus)、李斯特菌(Listeria monocytogenes)和 沙门氏菌等多种病原菌 ${ }^{[54]}$.

\section{2 竞争黏附位点, 增强肠道屏障功能}

竞争排斥是益生菌防止病原体入侵的能力之一, 通过与外源病原体竞争黏附空间导致病原体不能黏附 于肠腔, 从而防止病原菌进一步损害肠道屏障. 益生菌

表 3 合生元在母猪及仔猪中应用

Table 3 The effect of synbiotics on sows and piglets

\begin{tabular}{|c|c|c|c|c|}
\hline 合生元 & 剂量 & 实验对象 & 合生元的功能 & 参考文献 \\
\hline $\mathrm{scFOS}+\mathrm{LGG}$ & $\begin{array}{c}10 \mathrm{~g} / \mathrm{L} \mathrm{scFOS} \\
1 \times 10^{9} \text { CFU LGG }\end{array}$ & $1 \sim 8$ 天仔猪 & $\begin{array}{c}\text { 增加回肠黏膜重量、蛋白含量和线毛高度; 增 } \\
\text { 加表皮细胞增殖 }\end{array}$ & [44] \\
\hline Lactulose + L. plantarum & $\begin{array}{c}10 \mathrm{~g} / \mathrm{KG} \text { LAC } \\
2 \times 10^{9} \mathrm{CFU} \text { L. plantarum }\end{array}$ & 23 43天仔猪 & $\begin{array}{c}\text { 增加日增重; 增加回肠线毛高度; 增加结肠内乳 } \\
\text { 杆菌含量和丁酸浓度盐; 降低Pig-MAP; 降低腹 } \\
\text { 泻; 降低回肠和结肠食糜的氨浓度; 增加回肠的 } \\
\text { 杯状细胞数量; 降低TNF- } \alpha \text { 表达 }\end{array}$ & [45] \\
\hline Lactulose + E. faecium & $\begin{array}{c}5 \mathrm{~g} / \mathrm{KG} \mathrm{LAC} \\
10^{9} \mathrm{CFU} / \mathrm{KG} \mathrm{E} \text {. faecium }\end{array}$ & 4 8周仔猪 & $\begin{array}{c}\text { 厚壁菌门和拟杆菌门增加; 降低变形杆菌门降 } \\
\text { 低肠杆菌科; 增加乳杆菌属 }\end{array}$ & {$[46]$} \\
\hline 低聚果糖+益生菌 & $0.2 \% \mathrm{OF}$ 和 $0.3 \%$ 复合益生菌 & 母猪和其仔猪 & $\begin{array}{c}\text { 平均日增重增加; 降低结肠大肠杆菌; 增加回肠 } \\
\text { 双歧杆菌数量; }\end{array}$ & [47] \\
\hline 菊粉+复合益生菌 & $0.4 \%$ 菊粉和 $10^{9} \mathrm{CFU}$ 复合益生菌 & 26 54天仔猪 & $\begin{array}{l}\text { 增加空肠线毛高度; 下调TNF- } \alpha \text { 表达; } \\
\text { 下调NF-kB; 不影响血液指标; }\end{array}$ & [48] \\
\hline 菊粉+复合益生菌 & $0.4 \%$ 菊粉和 $10^{9} \mathrm{CFU}$ 复合益生菌 & 4 8周仔猪 & $\begin{array}{c}\text { 增加胃的干物质含量, 降低结肠干物质含量; 降 } \\
\text { 低回肠pH; 降低结肠内肠杆菌的数量 }\end{array}$ & [49] \\
\hline 菊粉+复合益生菌 & $0.4 \%$ 菊粉和 $10^{9} \mathrm{CFU}$ 复合益生菌 & 26 天仔猪 & 降低大肠杆菌数量; 增加双歧杆菌数量 & {$[50]$} \\
\hline
\end{tabular}


通过两种方式排斥有害菌: ( i ) 竞争营养吸收位点, 阻 止病原菌在肠道环境的增殖; (ii) 益生菌定殖后产生 溶菌酶或者过氧化氢等抑菌或者杀菌物质影响病原菌 的生存环境 ${ }^{[5]}$. 一些肠道病原菌可以利用鞭毛黏附到 宿主的表皮细胞，鞭毛的受体是甘露糖，添加酵母益 生菌可使病原菌凝集于含有 $D$-甘露糖的酵母细胞壁, 从而起到保护作用 ${ }^{[25]}$. Quilodrán-Vega等人 ${ }^{[56]}$ 发现弯 曲乳杆菌 (L. curvatus) 可以通过竞争和置换细胞黏附 位点的方式降低鼠伤寒沙门氏菌定殖在小鼠体内的数 量. 益生菌还可以通过调节细胞骨架和紧密连接蛋白 磷酸化来增强肠屏障功能 ${ }^{[57]}$. 植物乳杆菌 (L. plantar$u m$ )可以增加HT-29细胞系的MUC2 和MU3C表达，起 到保护肠道细胞的作用 ${ }^{[58]}$. 罗伊氏乳杆菌 (L. reuteri) I5007可增加新生仔猪紧密连接蛋白(tight junctions, $\mathrm{TJ})$ 的表达，在体外该菌株还可以抑制LPS诱导的TJ蛋 白表达的减少，表明罗伊氏乳杆菌I5007可能在TJ缺陷 条件下保护TJ蛋白, 从而维护肠道屏障的完整性 ${ }^{[28]}$.

\section{3 保护肠道形态}

线毛高度和隐窝深度是肠道成熟度和发挥功能的 间接指标. 在断奶时，小肠组织和形态会发生许多变 化, 如线毛高度减少(线毛萎缩)、隐窝深度增加(隐窝 增生 ${ }^{[59]}$. 线毛萎缩是由于线毛顶端细胞丢失率增加或 者细胞更新率降低. Bontepmo等人 ${ }^{[60]}$ 发现饲喂酵母菌 可以增加仔猪的线毛高度，降低线毛高度/隐窝深度的 比值. 短链脂肪酸是结肠微生物消化的主要代谢物, 其 中丁酸除了对结肠黏膜具有营养作用之外，还可为结 肠上皮细胞提供能量并调节细胞生长和分化. 据报道, 乳果糖和植物乳杆菌可增加结肠乳杆菌的含量和丁酸 盐的浓度(对照组对照组7.1 (mol\%) 合生元处理组12 $(\mathrm{mol} \%))^{[45]}$, 从而对维持结肠形态和功能中有重要作 用. Barnes等人 ${ }^{[44]}$ 研究发现低聚果糖和鼠李糖乳杆菌 $\mathrm{LGG}$ 可以增加小肠线毛高度，从而增加仔猪的营养吸 收, 增强肠道免疫屏障.

\section{4 刺激机体免疫系统, 抑制炎症因子}

新生仔猪肠道黏膜的免疫系统在出生后的前几个 星期发育不完善, 特别是肠道黏膜的 $\mathrm{T}$ 细胞成分, 需要 进行扩张和分化. 益生菌可刺激宿主免疫系统, 主要功 能包括: (i ) 增加吞噬能力; (ii) 增加NK细胞活力; (iii) 刺激IgA的产生; (iv) 抑制淋巴细胞增殖, 诱导细
胞调亡; (v) 增加细胞介导的免疫等 ${ }^{[61]}$. 如益生乳杆菌 可以调节许多炎症因子的表达，清酒乳杆菌(L.sakei) 可以降低IL-1 $\beta$, IL- 8 和TNF- $\alpha$ 的表达, 约氏乳杆菌 $(L$. johnsonii) 可以降低TGF- $\beta$ 的表达等 ${ }^{[62]}$. 饲喂乳双歧杆 菌, 可以增强血液白细胞吞噬能力以及 $\mathrm{T}$ 淋巴细胞增 殖能力, 同时提高胃肠道病原菌的特异性抗体滴度, 从而减少由轮状病毒和大肠杆菌引起的仔猪腹泻 ${ }^{[63]}$. 母猪的健康水平与仔猪的出生体重和存活率密切相 关 ${ }^{[64]}$. 益生元可以通过改善母猪肠道健康状态提高仔 猪健康水平, 如Le Bourgot等人 ${ }^{[39]}$ 研究发现在妊娠期 和哺乳期母猪饲料中添加 scFOS 可以提高初乳中 IgA 和TGF- $\beta 1$ 水平, 增加仔猪潘氏细胞和肠系腹淋巴结分 泌的IFN- $\gamma$ 和SIgA增加, 从而促进仔猪肠道免疫系统的 成熟. Mair等人 ${ }^{[48]}$ 研究发现添加菊粉和复合益生菌(乳 杆菌、粪肠球菌和双歧杆菌), 可下调炎症标记基因 TNF- $\alpha$ 和NF- $\kappa B$ 的表达.

\section{5 调节肠道菌群}

猪的肠道微生物菌群对身体健康和营养吸收起着 非常重要的作用. 许多研究表明, 仔猪断奶期间肠道微 生物多样性降低, 其中专性厌氧菌(如梭菌和拟杆菌) 显著降低, 乳杆菌属的数量显著减少, 兼性厌氧菌(如 大肠杆菌)的数量相对增加. 大肠杆菌代谢氨基酸过程 中脱羧而生的胺可刺激肠道导致腹泻，从而对宿主产 生毒性作用 ${ }^{[25]}$. 同时, 微生态失调将导致肠道紊乱和 胃肠道感染 ${ }^{[10]}$. 益生菌可增加肠道微生物多样性, 通 过增加厚壁菌门中乳杆菌属和双歧杆菌的数量, 降低 幽门螺杆菌和肠杆菌等潜在病原菌数量, 调节肠道微 生物的组成, 从而改善仔猪肠道健康, 降低腹江率. Jiao等人 ${ }^{[40]}$ 研究发现, 添加纤维素寡糖可以影响断奶 仔猪肠道微生物群, 使乳杆菌属的丰度增加, 降低潜 在有害菌(梭菌属和肠杆菌属)的数量. Paßlack等人 ${ }^{[42]}$ 研究发现对妊娠期和哺乳期母猪饲喂菊粉可以增加母 猪粪便中肠球菌的数量, 增加仔猪盲肠内肠球菌数量, 增加仔猪胃内真菌和盲肠中柔嫩梭菌的数量, 降低仔 猪胃中肠杆菌和食淀粉乳杆菌的数量, 由此调节仔猪 肠道健康. Guerra-Ordaz等人 ${ }^{[45]}$ 研究发现添加乳果糖 和植物乳杆菌可降低肠杆菌的数量, 从而调节肠道微 生物群, 减少断奶仔猪的腹泻率. 低聚果糖复合益生 菌、菊粉复合益生菌以及乳果糖和粪肠球菌复合, 也 能够增加乳杆菌或双歧杆菌的数量, 降低变形菌门或 


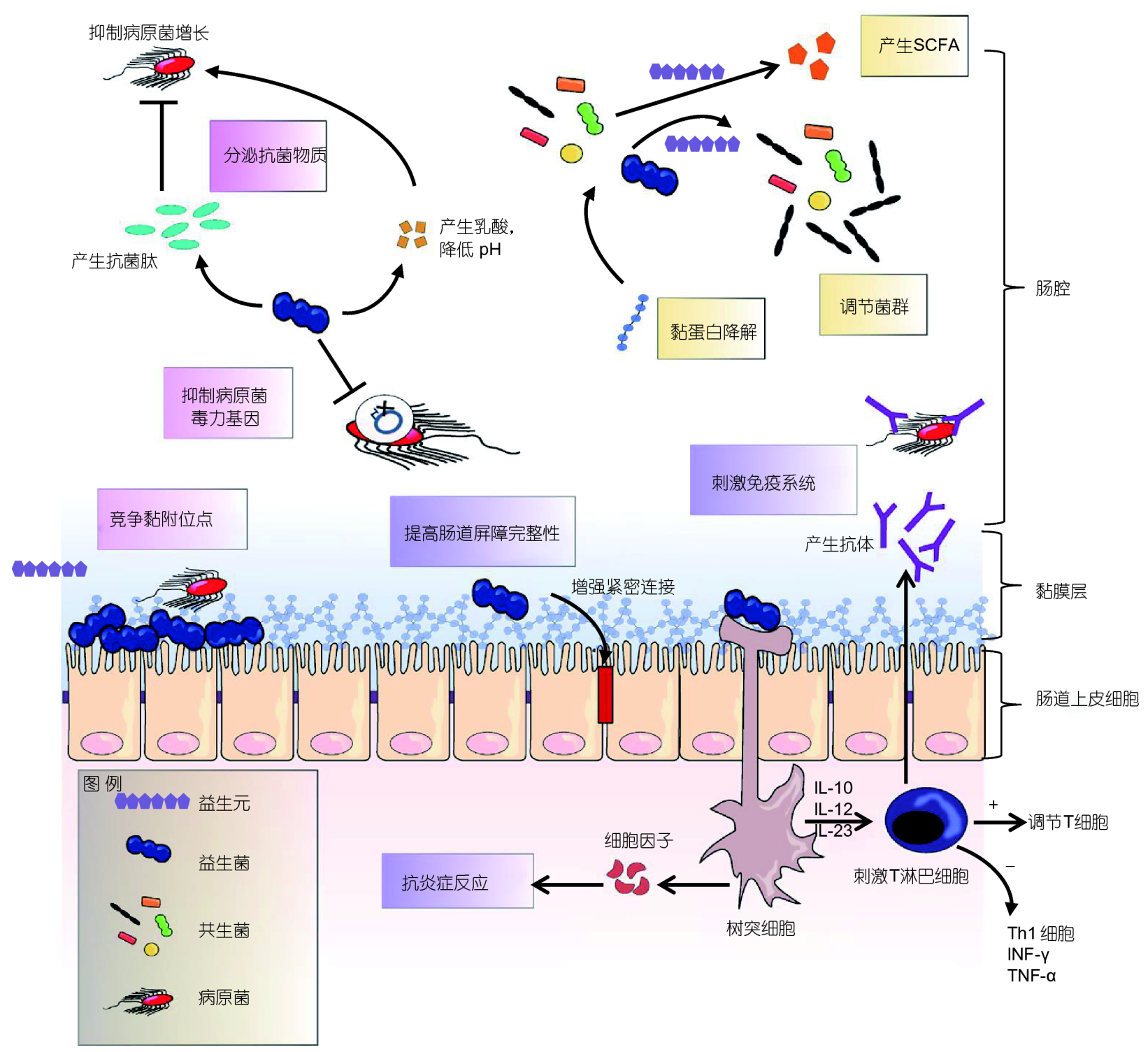

图 1 益生菌和益生元改善肠道健康的功能机制. ( i ) 益生菌通过产生有机酸或者抗菌物质等抑制病原菌的生长; (ii) 益生 菌通过直接与病原菌作用抑制病原菌毒力因子的表达; (iii) 益生菌直接产生短链脂肪酸, 益生菌利用低聚糖等益生元产生短 链脂肪酸, 共生菌利用低聚糖等益生元产生短链脂肪酸; (iv) 益生菌调节土著菌群, 维持菌群的多样性和稳定性, 产生有益于 宿主的物质; (v) 益生菌竞争排斥病原菌, 防止病原菌的入侵, 益生元被益生菌利用, 增加益生菌的数量; (vi) 益生菌刺激紧密 连接蛋白的表达, 增强肠道黏膜屏障; (vii) 益生菌通过PAMP与树突细胞toll样受体结合刺激树突细胞发挥免疫效应; (viii) T细 胞通过分化调节 $\mathrm{T}$ 细胞和辅助 $\mathrm{T}$ 细胞发挥免疫功能; (ix) 树突细胞通过抗炎症因子 IL-10等调节 $\mathrm{T}$ 细胞产生抗体与病原体抗原结 合, 消灭病原菌; ( $\mathrm{x}$ ) 树突细胞通过产生细胞因子发挥抗炎症作用 (该图结合了体外和体内的实验数据 ${ }^{[8,35]}$ ) (网络版彩图)

Figure 1 Functional mechanisms of probiotics and prebiotics for improving intestinal health. (i) Probiotics could inhibit the growth of pathogenic bacteria by producing organic acids or antibacterial substances; (ii) Probiotics could inhibit the expression of pathogenic virulence factors through interacting with pathogenic bacteria; (iii) Probiotics could produce short-chain fatty acids directly or by using prebiotics, such as oligosaccharides. Symbiotic bacteria could use prebiotics such as oligosaccharides to produce short-chain fatty acids; (iv) Probiotics could modulate the resident microbiota composition and maintain the diversity and stability of the microbiota, and produce substances that are beneficial to the host; (v) Probiotics could compete against pathogens and prevent the invasion of pathogens. Prebiotics are utilized by probiotics to increase the number of probiotics; (vi) Probiotics could stimulate the expression of tight junction proteins and enhance the intestinal mucosal barrier; (vii) Probiotics could bind to toll-like receptors to exert immune effects via a pathogen associated molecular pattern (PAMP); (viii) T cells could regulate immune function via cell differentiation regulatory $\mathrm{T}$ cells and helper T cells; (ix) Dendritic cells could regulate $\mathrm{T}$ cells via anti-inflammatory factors such as IL-10 to produce antigens which bind to pathogens to eliminate them; (x) Dendritic cells exert anti-inflammatory effects by producing cytokines. (This figure combines experimental data in vitro and in vivo ${ }^{[8,35]}$ (color online) 
大肠杆菌的数量, 从而调节肠道菌群 ${ }^{[45,49,50]}$. 值得注意 的是, 通常情况下合生元中益生菌和益生元的作用具 有协同作用，如菊粉和复合益生菌(Enterococcus faecium, L. salivarius, L. reuteri, Bifidobacterium thermophilum)组成的合生元饲喂断奶仔猪，其肠道中的粪肠 球菌数量多于菊粉和复合益生菌单独添加组，这说明 菊粉可以促进复合益生元中粪肠球菌的增长，达到协 同作用, 稳定益生效果 ${ }^{[49]}$. 同样, 低聚果糖和鼠李糖乳 杆菌复配的合生元可促进仔猪回肠线毛高度，其效果 强于单独添加低聚果糖或鼠李糖乳杆菌组 ${ }^{[4]}$.

\section{4 展望}

断奶应激一直是困扰养猪业的一个难题，也是动 物营养学界亟待解决的课题之一. 由于幼龄仔猪消化 系统和免疫系统都尚未发育成熟，容易受到病原菌入 侵，早期断奶还容易造成仔猪胃肠道功能紊乱，诱发 仔猪断奶综合征 ${ }^{[65]}$. 随着抗生素耐药菌日益增多, 尤
其是耐受多黏菌素的基因 $(m c r-1)$ 在人体和动物的肠 道泛滥, 标志着人体最后一道防线被耐药菌突破 ${ }^{[3,66]}$, 建立并研发能够调节仔猪肠道健康的安全有效的抗生 素替代物十分必要. 微生态制剂有着不可替代的优点, 其“患病治病，未病防病，无病保健”的效果，避免了抗 生素长期使用带来的毒副作用、细菌耐药性和抗药性. 微生态制剂微生态制剂通过多种方式改善仔猪肠道健 康, 包括促进小肠发育, 增加营养的吸收; 增加结肠内 短链脂肪酸浓度, 降低 $\mathrm{pH}$, 分泌抑菌物质, 抵抗病原菌; 提高紧密连接蛋白的表达、增强黏膜屏障; 调节肠道 菌群平衡; 提高免疫力, 降低炎症因子等(图1). 随着组 学技术的发展, 未来宏基因组、宏转录组、代谢组等 多组学分析手段也利于我们了解微生态制剂在肠道微 生态中的特异作用，如营养物质摄入后如何被肠道微 生物利用 ${ }^{[67]}$, 产生生物活性代谢物有什么, 它们如何影 响宿主的调节功能等 ${ }^{[68]}$. 而对特定益生元的特殊益生 作用、益生元和益生菌的真实使用功效和他们在腹泻 仔猪中的使用剂量等也都需要更深入的探索和研究.

\section{参考文献}

1 Huang M, Liu Y, Wang Hui, et al. Progress on etiology of piglet diarrhea (in Chinese). Prog Veter Med, 2015, 36: 88-91 [黄美州, 刘永明, 王慧, 等. 仔猪腹泻病原学研究进展. 动物医学进展, 2015, 36: 88-91]

2 Chen X, Zhao L, Gao S, et al. Virulence genes of pathogenic Escherichia coli from ill pigs in China and their relationship with O-serogroups (in Chinese). Acta Microbiol Sin, 2008, 48: 857-862 [陈祥, 赵李祥, 高崧, 等. 猪源大肠杆菌(ETEC, STEC, AEEC)毒力基因及其与O抗原型的关 系. 微生物学报, 2008, 48: 857-862]

3 Liu Y Y, Wang Y, Walsh T R, et al. Emergence of plasmid-mediated colistin resistance mechanism MCR-1 in animals and human beings in China: a microbiological and molecular biological study. Lancet Infect Dis, 2016, 16: 161-168

$4 \mathrm{He}$ S, Wang Q, Li S, et al. Antibiotic growth promoter olaquindox increases pathogen susceptibility in fish by inducing gut microbiota dysbiosis. Sci China Life Sci, 2017, 60: 1260-1270

5 Jensen J, Kyvsgaard N C, Battisti A, et al. Environmental and public health related risk of veterinary zinc in pig production-using Denmark as an example. Environ Int, 2018, 114: 181-190

6 Sutherland M A, Backus B L, McGlone J J. Effects of transport at weaning on the behavior, physiology and performance of pigs. Animals, 2014, 4: 657-669

7 Lallès J P, Bosi P, Smidt H, et al. Nutritional management of gut health in pigs around weaning. Proc Nutr Soc, 2007, 66: 260-268

8 Ma Q, Yu Z, Zhang R. Lactobacillus preparation and its application in maintaining intestinal health of weaned piglets (in Chinese). Feed Ind, 2010, 31: 10-12 [马青山, 余占桥, 张日俊. 乳酸杆菌制剂及其在维护断奶仔猪肠道健康中的应用. 饲料工业, 2010, 31: 10-12]

9 Melotti L, Oostindjer M, Bolhuis J E, et al. Coping personality type and environmental enrichment affect aggression at weaning in pigs. Appl Animal Behaviour Sci, 2011, 133: 144-153

10 Gresse R, Chaucheyras-Durand F, Fleury M A, et al. Gut microbiota dysbiosis in postweaning piglets: understanding the keys to health. Trends Microbiol, 2017, 25: 851-873

11 Lallès J P, Bosi P, Smidt H, et al. Weaning - a challenge to gut physiologists. Livestock Sci, 2007, 108: 82-93

12 Isaacson R, Kim H B. The intestinal microbiome of the pig. Anim Health Res Rev, 2012, 13: 100-109 
13 Petri D, Hill J E, Van Kessel A G. Microbial succession in the gastrointestinal tract (GIT) of the preweaned pig. Livestock Sci, 2010, 133: 107109

14 Bian G, Ma S, Zhu Z, et al. Age, introduction of solid feed and weaning are more important determinants of gut bacterial succession in piglets than breed and nursing mother as revealed by a reciprocal cross-fostering model. Environ Microbiol, 2016, 18: 1566-1577

15 Bäumler A J, Sperandio V. Interactions between the microbiota and pathogenic bacteria in the gut. Nature, 2016, 535: 85-93

Zhou P, Fan H, Lan T, et al. Fatal swine acute diarrhoea syndrome caused by an HKU2-related coronavirus of bat origin. Nature, 2018, 556: 255258

17 Saif L J. Coronavirus immunogens. Veterinary MicroBiol, 1993, 37: 285-297

18 Pospischil A, Hou Y, Qi C. Progress in research on swine viral diarrhea (in Chinese). Foreign Anim Husb Technol, 1990, 4: 47-49 [Pospischil A, 侯引绪, 齐长明. 猪病毒性腹泻研究进展. 国外畜牧科技, 1990, 4: 47-49]

19 Tran T H T, Everaert N, Bindelle J. Review on the effects of potential prebiotics on controlling intestinal enteropathogens Salmonella and Escherichia coli in pig production. J Anim Physiol Anim Nutr, 2018, 102: 17-32

20 Kim H B, Isaacson R E. Salmonella in swine: microbiota interactions. Annu Rev Anim Biosci, 2017, 5: 43-63

21 Toutain P L, Ferran A A, Bousquet-Melou A, et al. Veterinary medicine needs new green antimicrobial drugs. Front Microbiol, 2016, 7: 1196

22 Hempel S, Newberry S J, Maher A R, et al. Probiotics for the prevention and treatment of antibiotic-associated diarrhea. JAMA, 2012, $307:$ 1959

Parker RB. Probiotics-The other half of the antibiotics story. Anim Nutr Health, 1974, 29: 4-8

Patil A, Kumar S, Verma AK, et al. Probiotics as feed additives in weaned pigs: a review. Livest Res Int, 2015, 3: 31-39

Cofichs MD, Gibson GR. Probiotics, prebiotics, and synbiotics: approaches for modulating the microbial ecology of the gut. Am J Clin Nutr, 1999, 69: 1052s-1057s

27 Liu H, Hou C, Wang G, et al. Lactobacillus reuteri I5007 modulates intestinal host defense peptide expression in the model of IPEC-J2 cells and neonatal piglets. Nutrients, 2017, 9: 559

28 Yang F, Wang A, Zeng X, et al. Lactobacillus reuteri I5007 modulates tight junction protein expression in IPEC-J2 cells with LPS stimulation and in newborn piglets under normal conditions. BMC Microbiol, 2015, 15: 32

29 Brousseau J P, Talbot G, Beaudoin F, et al. Effects of probiotics Pediococcus acidilactici strain MA18/5M and Saccharomyces cerevisiae subsp. boulardii strain SB-CNCM I-1079 on fecal and intestinal microbiota of nursing and weanling piglets1. J Anim Sci, 2015, 93: 5313-5326

30 Dowarah R, Verma A K, Agarwal N, et al. Effect of swine based probiotic on performance, diarrhoea scores, intestinal microbiota and gut health of grower-finisher crossbred pigs. Livestock Sci, 2017, 195: 74-79

31 Riboulet-Bisson E, Sturme M H J, Jeffery I B, et al. Effect of Lactobacillus salivarius bacteriocin Abp118 on the mouse and pig intestinal microbiota. PLoS ONE, 2012, 7: e31113

$32 \mathrm{Hu} \mathrm{Y}$, Dun Y, Li S, et al. Dietary Enterococcus faecalis LAB31 improves growth performance, reduces diarrhea, and increases fecal Lactobacillus number of weaned piglets. PLoS ONE, 2015, 10: e0116635

33 Konstantinov S R, Smidt H, Akkermans A D L, et al. Feeding of Lactobacillus sobrius reduces Escherichia coli F4 levels in the gut and promotes growth of infected piglets. FEMS Microbiol Ecol, 2008, 66: 599-607

34 Zhang L, Xu Y Q, Liu H Y, et al. Evaluation of Lactobacillus rhamnosusGG using an Escherichia coli K88 model of piglet diarrhoea: effects on diarrhoea incidence, faecal microflora and immune responses. Veterinary Microbiol, 2010, 141: 142-148

35 Barba-Vidal E, Castillejos L, Roll V F B, et al. The probiotic combination of Bifidobacterium longum subsp. infantis CECT 7210 and Bifidobacterium animalis subsp. lactis BPL6 reduces pathogen loads and improves gut health of weaned piglets orally challenged with Salmonella typhimurium. Front Microbiol, 2017, 8: 1570

36 Yang G Y, Zhu Y H, Zhang W, et al. Influence of orally fed a select mixture of Bacillus probiotics on intestinal T-cell migration in weaned MUC4 resistant pigs following Escherichia coli challenge. Vet Res, 2016, 47: 71

37 Trevisi P, Latorre R, Priori D, et al. Effect of feed supplementation with live yeast on the intestinal transcriptome profile of weaning pigs orally challenged with Escherichia coli F4. Animal, 2017, 11: 33-44

38 Ducatelle R, Eeckhaut V, Haesebrouck F, et al. A review on prebiotics and probiotics for the control of dysbiosis: present status and future perspectives. Animal, 2015, 9: 43-48

39 Le Bourgot C, Ferret-Bernard S, Le Normand L, et al. Maternal short-chain fructooligosaccharide supplementation influences intestinal immune 
system maturation in piglets. PLoS ONE, 2014, 9: e107508

40 Jiao L F, Song Z H, Ke Y L, et al. Cello-oligosaccharide influences intestinal microflora, mucosal architecture and nutrient transport in weaned pigs. Anim Feed Sci Tech, 2014, 195: 85-91

41 Castillo M, Martin-Orue S M, Taylor-Pickard J A, et al. Use of mannanoligosaccharides and zinc chelate as growth promoters and diarrhea preventative in weaning pigs: effects on microbiota and gut function1. J Anim Sci, 2008, 86: 94-101

42 Paßlack N, Vahjen W, Zentek J. Dietary inulin affects the intestinal microbiota in sows and their suckling piglets. BMC Vet Res, 2015, 11: 51

43 Letellier A, Messier S, Lessard L, et al. Assessment of various treatments to reduce carriage of Salmonella in swine. Can J Veter Res, 2000, 64: 27

44 Barnes J L, Hartmann B, Holst J J, et al. Intestinal adaptation is stimulated by partial enteral nutrition supplemented with the prebiotic short-chain fructooligosaccharide in a neonatal intestinal failure piglet model. JPEN J Parenter Enteral Nutr, 2012, 36: 524-537

45 Guerra-Ordaz A A, González-Ortiz G, La Ragione R M, et al. Lactulose and Lactobacillus plantarum, a potential complementary synbiotic to control postweaning colibacillosis in piglets. Appl Environ Microbiol, 2014, 80: 4879-4886

46 Chae J P, Pajarillo E A B, Oh J K, et al. Revealing the combined effects of lactulose and probiotic enterococci on the swine faecal microbiota using 454 pyrosequencing. Microb Biotechnol, 2016, 9: 486-495

47 Shim S B, Verstegen M W A, Kim I H, et al. Effects of feeding antibiotic-free creep feed supplemented with oligofructose, probiotics or synbiotics to suckling piglets increases the preweaning weight gain and composition of intestinal microbiota. Archiv Anim Nutr, 2005, 59: 419427

48 Mair C, Plitzner C, Pfaffl M W, et al. Inulin and probiotics in newly weaned piglets: effects on intestinal morphology, mRNA expression levels of inflammatory marker genes and haematology. Archiv Anim Nutr, 2010, 64: 304-321

49 Mair C, Plitzner C, Domig K J, et al. Impact of inulin and a multispecies probiotic formulation on performance, microbial ecology and concomitant fermentation patterns in newly weaned piglets. J Anim Physiol Anim Nutr, 2010, 94: e164-e177

50 Sattler V A, Bayer K, Schatzmayr G, et al. Impact of a probiotic, inulin, or their combination on the piglets' microbiota at different intestinal locations. Beneficial Microbes, 2015, 6: 473-483

51 Asahara T, Shimizu K, Nomoto K, et al. Probiotic Bifidobacteria protect mice from lethal infection with Shiga toxin-producing Escherichia coli O157:H7. Infection Immun, 2004, 72: 2240-2247

52 Pridmore R D, Pittet A C, Praplan F, et al. Hydrogen peroxide production by Lactobacillus johnsonii NCC 533 and its role in anti-Salmonella activity. FEMS Microbiol Lett, 2008, 283: 210-215

53 Cintas L M, Casaus M P, Herranz C, et al. Review: bacteriocins of lactic acid bacteria. Food Sci Technol Int, 2001, 7: 281-305

54 Flynn S, van Sinderen D, Thornton G M, et al. Characterization of the genetic locus responsible for the production of ABP-118, a novel bacteriocin produced by the probiotic bacterium Lactobacillus salivarius subsp. salivarius UCC118. Microbiology, 2002, 176: 973-984 Brown M. Modes of action of probiotics: recent developments. J An Veter Adv, 2011, 10: 1895-1900 Quilodrán-Vega S R, Villena J, Valdebenito J, et al. Isolation of lactic acid bacteria from swine milk and characterization of potential probiotic strains with antagonistic effects against swine-associated gastrointestinal pathogens. Can J Microbiol, 2016, 62: 514-524

57 Sherman P M, Johnson-Henry K C, Yeung H P, et al. Probiotics reduce enterohemorrhagic Escherichia coli O157:H7- and enteropathogenic E. coli $\mathrm{O} 127$ :H6-induced changes in polarized T84 epithelial cell monolayers by reducing bacterial adhesion and cytoskeletal rearrangements. Infect Immun, 2005, 73: 5183-5188

58 Ng S C, Hart A L, Kamm M A, et al. Mechanisms of action of probiotics: recent advances. Inflamm Bowel Dis, 2009, 15: 300-310

59 Hampson D. Alteration in piglet small intestinal structure at weaning. 1986, 40: 32-40

60 Bontempo V, Di Giancamillo A, Savoini G, et al. Live yeast dietary supplementation acts upon intestinal morpho-functional aspects and growth in weanling piglets. Anim Feed Sci Tech, 2006, 129: 224-236

61 Delcenserie V, Martel D, Lamoureux M, et al. Immunomodulatory effects of probiotics in the intestinal tract. Curr Issues Mol Biol, 2008, 10: 3754

62 Delcenserie V, Martel D, Lamoureux M, et al. Immunomodulatory effects of probiotics in the intestinal tract. FEMS Microbiol Lett, 2008, 10: 3754

63 Shu Q, Qu F, Gill H S. Probiotic treatment using Bifidobacterium lactis HN019 reduces weanling diarrhea associated with rotavirus and Escherichia coli infection in a piglet model. J Pediatr Gastroenterol Nutr, 2001, 33: 171-177 
64 Bin P, Azad M A K, Liu G, et al. Effects of different levels of methionine on sow health and plasma metabolomics during late gestation. Food Funct, 2018, 9: 4979-4988

65 Yin Y, Li T, Wu X, et al. Research and application of biological mechanisms and regulation technique in intestinal health of piglets (in Chinese). Chin J Anim Nutr, 2010, 22: 10-17 [印遇龙, 李铁军, 吴信, 等. 仔猪肠道健康的生物学机制及调控技术研究与应用. 动物营养学报, 2010, 22: 10-17]

66 Li Z, Tan C, Lin J, et al. Diversified variants of the mcr-1-carrying plasmid reservoir in the swine lung microbiota. Sci China Life Sci, 2016, 59: 971-973

67 Chen S, Wang M, Yin L, et al. Effects of dietary tryptophan supplementation in the acetic acid-induced colitis mouse model. Food Funct, 2018, 9: $4143-4152$

68 Valeriano V D V, Balolong M P, Kang D K. Probiotic roles of Lactobacillus sp. in swine: insights from gut microbiota. J Appl Microbiol, 2017, 122: $554-567$

\title{
The functions and mechanisms of microecological agents in weaning piglet intestine
}

\author{
WANG TianWei ${ }^{1,2 \dagger}$, TENG KunLing ${ }^{1 \dagger}$, LIU Gang ${ }^{3}$, LIU YaYong ${ }^{1,2}$, ZHANG Xin $^{4}$, DONG EnQiu ${ }^{4}$, \\ YIN YuLong ${ }^{3} \&$ ZHONG Jin ${ }^{1,2^{*}}$ \\ 1 Institute of Microbiology, Chinese Academy of Sciences, Beijing 100101, China; \\ 2 University of Chinese Academy of Sciences, Beijing 100101, China; \\ 3 Institute of Subtropical Agriculture, Chinese Academy of Sciences, Changsha 410125, China; \\ 4 LongDa Foodstuff Group Co., Ltd, Yantai 265231, China
}

The high mortality rate of weaning piglets caused by diarrhea is an important issue that hinders the development of pig industry. Weaning piglets are susceptible to pathogenic bacteria, viruses and other stresses which lead to gastrointestinal dysfunction due to their immature intestinal barrier and immune system. Antibiotics can increase economic efficiency by improving the status of animal husbandry, however, the abuse of antibiotics has led to the emergence of antibiotic resistant strains, which seriously injured the human health. As a result, it is urgent to develop safe alternatives to reduce or replace antibiotics. Microecological agents are safe, drugresistance-free and have non-toxic side effects and positive impact on intestinal health of animals. Therefore, understanding the mechanisms of microecological agents in improving intestinal health has important implications for the effective use of them. This review summarizes the cause of diarrhea and the major probiotic functions and mechanisms of microecological agents in weaning piglets.

microecological agents, weaning piglets, intestinal health, mechanisms

doi: $10.1360 / \mathrm{N} 052018-00143$ 